\title{
Laser for Wakefield Plasma Accelerator
}

\author{
by \\ Gérard Mourou \\ Director, Center for Ultrafast Optical Science, \\ Professor of Electrical Engineering and Computer Science, University of Michigan
}

Over the past ten years a revolution has occurred in our ability to produce high-peak-power pulses (Fig. 1). Ten years ago, tabletop-size lasers could generate only gigawatt power. Today, the same size system can produce at least a thousand times this power, i.e., terawatt. This dramatic advance has been due to the technique of Chirped Pulse Amplification (CPA) ${ }^{1}$ and our ability to produce short pulses. ${ }^{2}$ Once focused, intensities over $10^{18} \mathrm{~W} / \mathrm{cm}^{2}$ can easily be achieved (Fig. 2). At these intensities the electron quiver velocity is close to the speed of light and the electron mass changes periodically in the field of the laser. This relativistic optical regime opens novel and attractive opportunities, particularly in the field of laser plasma accelerators.

In 1979, T. Tajima and J. M. Dawson proposed a plasma-based accelerator concept called wakefield accelerator. ${ }^{3}$ In this concept, a very intense pulse propagates into a subcritical-density plasma to create a large plasma wave in its wake. The electrostatic gradient associated with the wave is very large. For the TableTop Terawatt Laser $\left(\mathrm{T}^{3}\right)$ the magnitude of this gradient is of the order of $20 \mathrm{GV} / \mathrm{m}$, that is, $10^{3}$ times greater than the gradient obtained by conventional methods. ${ }^{4}$ It is important to note that this technique is similar to the Beat Wave Accelerator, ${ }^{3}$ where instead of using one pulse to create the plasma, a series of pulses generated by beating two waves is used. The difference frequency is set to be equal to the plasma frequency which can be adjusted by changing the plasma density. This concept has, however, a limitation. As the electrons get accelerated their mass changes, so the interval between the pulses needs to be readjusted in order for the particles to stay in phase with the plasma wave. This condition cannot be met with the beat wave, where the pulse separation is rigidly fixed. To accommodate for the relativistic detuning a third concept, Resonantly Laser Driven Plasma Accelerator (RLPA), was proposed by D. Umstadter and E. Esarey, ${ }^{5}$ with the plasma wave this time driven by a series of pulses, where the distance between the pulses changes to take into account the change in the plasma frequency due to the increase in the electron mass. RLPA leads to a much-improved efficiency, 5

Another important consideration for laser accelerators is the average current or power. For a tera-electron-volt-size accelerator the average power is in the $10-\mathrm{kW}$ range. The laser average power of current CPA systems is in the 1-W range. To be competitive with existing technology, laser power in the kilowatt range will have to become available.

We can at this point list the desired characteristics for a laser driver compatible with the acceleration of electrons in the tera-electron-volt range:

1. Intensities greater than $10^{18} \mathrm{~W} / \mathrm{cm}^{2}$ over a large spot size, typically greater than $50 \mu \mathrm{m}$, corresponding to several plasma wavelengths to avoid diffraction (1-D regime).

2. Adjustable pulse duration from $50 \mathrm{fs}$ to 500 fs for optimum performance. 
3. Pulse shaping capability. To avoid relativistic detuning, a sequence of pulses will drive the plasma. Both pulse duration and separation will have to be adjusted with femtosecond accuracy.

4. High average power greater than $1 \mathrm{~kW}$.

5. Low cost. For high-average-power, tera-electron-volt, multiple synchronized beams, as many as 1000 could be required.

We will in the following section discuss these different points essential to the construction of a compact laser wakefield accelerator.

\section{Ultrahigh Intensity}

The concept of Chirped Pulse Amplification is described in Fig. 4. A good energy storage material, i.e., with a small transition cross section and wide bandwidth, is used to amplify a short-duration seed pulse. In order to circumvent the nonlinear effects taking place in the optical elements i.e., small-scale self-focusing as the pulse is amplified, the pulse is stretched $10^{3}$ to $10^{4}$ times, reducing the intensity by $10^{3}$ to $10^{4}$ without changing the input fluence $\left(\mathrm{J} / \mathrm{cm}^{2}\right)$. At this point the pulse can be amplified and efficiently extract the energy stored in the amplifying medium. After the energy is fully extracted the pulse is compressed to its initial value. The concept has been shown up to 80 TW6 $^{6}$ and further extension up to the petawatt ${ }^{7}$ will be demonstrated in the near future. Although a large improvement in peak power has been shown (see Fig. 1) over the last few years, we are still far from the ultimate peak power level of good energy storage materials like Ti:sapphire, alexandrite, Nd:glasses, and LiSAF. The theoretical peak power is given by the ratio between the saturation fluence over the reciprocal gain bandwidth $\mathrm{P}_{\mathrm{th}}=2 \mathrm{~F}_{\mathrm{sat}}$. Du, where, Fsat is the saturation fluence and $\mathrm{Du}$ is the gain bandwidth at half maximum. This value corresponds to the Rabi intensity to produce half-Rabi oscillation in these materials. These intensities are expressed below for beam size of $\mathrm{a} \mathrm{cm}^{2}$, for different amplifying media:

$\begin{array}{ll}\text { Nd:phosphate } & P_{\text {th }}=60 \mathrm{TW} / \mathrm{cm}^{2} \\ \text { Nd:silicate } & P_{\text {th }}=100 \mathrm{TW} / \mathrm{cm}^{2} \\ \text { Nd:glass combination } & P_{\text {th }}=400 \mathrm{TW} / \mathrm{cm}^{2} \\ \text { Alexandrite } & P_{\text {th }}=2000 \mathrm{TW} / \mathrm{cm}^{2} \\ \text { Ti:sapphire } & P_{\text {th }}=120 \mathrm{TW} / \mathrm{cm}^{2}\end{array}$

The saturation fluence of these materials being of the order of $0.5 \mathrm{~J} / \mathrm{cm}^{2}$ (Ti:sapphire) to 10 $\mathrm{J} / \mathrm{cm}^{2}$ (alexandrite), to prevent optical damage ${ }^{8}$, the pulse will have to be stretched from $10 \mathrm{fs}$ to greater than $1 \mathrm{~ns}$, i.e., greater than $10^{5}$ times. This expansion and compression ratio is more than 10 times what can be accomplished today. In the initial CPA work, ${ }^{1}$ a fiber was used to stretch and a grating pair to compress. Because the stretcher and compressor were not matched, the expansion compression was limited to about hundred. It was realized afterward that a gratingbased compressor proposed by $O$. Martinez ${ }^{9}$ for the compression of stretched pulses at $1500 \mathrm{~nm}$, i.e., in the negative group velocity dispersion of fiber, was matched with the grating compressor over all orders. Stretching/compression of a few thousand was demonstrated. ${ }^{10}$ This system now is used in all CPA amplifying systems. ${ }^{11}$ For very short pulse amplificaion, further improvements on the basic system were done by C. Barty 12 and by Mumane and Kapteyn. ${ }^{13}$ To 
reach the theoretical peak power limit, a $10^{5}$ time stretcher compressor system matched over all orders will have to be demonstrated and deployed. Important work in this area are being done by M. White et al. ${ }^{14}$ and S. Kane et al. 15

\section{Adjustable Pulse Duration for Optimum Performance}

In wakefield acceleration it is important that the pulse duration be of the order of the plasma period. It also determines the bucket structure where the electrons can be trapped and accelerated. The number of buckets is a function of how quickly the plasma wave can be damped. The optimum pulse duration is found ${ }^{16}$ to be of the order of $100 \mathrm{fs}$ and must be adjustable. It is one of the great features of CPA to permit the continuous variation of the pulse duration from femtoseconds to nanoseconds by a simple change in the compression grating distance, keeping all other parameters-energy, pulse bandwidth, beam quality-constant.

\section{Pulse Shaping Capability}

As discussed by $D$. Umstadter and Esarey, 5 it is more efficient to excite the plasma wave with a series of pulses in a prescribed sequence than with a single pulse. It is therefore essential to have pulse-shaping capability. This can be easily obtained using a pulse stacker approach, where the main beam is split in several beams which are then delayed with respect to each other and recombined. A more sophisticated approach consists of filtering in phase and amplitude, in the Fourier plane of the stretcher, the pulse Fourier components. ${ }^{17}$ Figure 5 shows some of the results obtained by using this technique.

\section{High Average Power}

High beam current will require a high-average-power laser. Current systems that provide intensities in the $10^{18}-\mathrm{W} / \mathrm{cm}^{2}$ range have average power of the order of one watt, 18 i.e., $100 \mathrm{~mJ}$ at $10 \mathrm{~Hz},{ }^{19}$ or $1 \mathrm{~J}$ at $1 \mathrm{~Hz} .{ }^{20}$ High-energy physics requires high average power in the range of 100 $\mathrm{kW}$. For laser-to-electron-beam efficiency of the order of $10 \%$, average laser power of the order of $10 \mathrm{~kW}$ will be necessary. Average power of the order of $50 \mathrm{~W} / \mathrm{cm}^{3}$ of optical amplifier has been demonstrated for laser-diode-pumped Nd:glass. ${ }^{21} \mathrm{~A} 10-\mathrm{kW}$-average-power laser, $\mathrm{Nd}$ :glasses, could be built with only $400 \mathrm{~cm}^{3}$ of $\mathrm{Nd}$ :glasses. To be efficient the laser will be continuously pumped at a repetition rate roughly equal to $1 / \mathrm{Tf}$, corresponding to $1 \mathrm{kHz}$ for Nd:glasses. The average energy per pulse will therefore be of the order of $10 \mathrm{~J}$ with a pulse width of $100 \mathrm{fs}$, i.e., a peak power of $100 \mathrm{TW}$. If we mix Nd:glasses to increase the gain bandwidth, pulses as short as $30 \mathrm{fs}$ could be produced. The overall characteristics of the system will be 100 TW at $1 \mathrm{kHz}$. It is also possible for this application to have several regenerative amplifiers as shown in Fig. 6. Each regenerative amplifier will excite a different plasma cell. A tera-electronvolt-class wakefield accelerator could involve as many as 1000 . plasma cells. Each plasma cell will be of the order of a centimeter. The overall accelerator length will be of the order of 10 to $100 \mathrm{~m}$ for a beam size of the order of $1 \mathrm{~cm}$. The level of complexity of such a system will be less than the large MJ fusion laser with 280 beams where each beam is $60 \mathrm{~cm}$ in diameter. 


\section{Low Cost}

The system could be quite economical. Nd:glass is inexpensive and well understood. The cost of a laser diode will decline in the near future. The system of choice will rely on large regenerative amplifiers capable of producing $10 \mathrm{~J}$ pulses at $1 \mathrm{kHz}$. Similar technology has been demonstrated in laser fusion; where large regenerative amplifiers involving large-aperture Pockels cells with transparent plasma electrodes are used for injection and cavity dumping.

\section{Conclusion}

From a laser point of view, the laser wakefield accelerator seems to be doable. Large peak power and large intensities can be produced to excite the plasma waves. The optimum duration of the order of $100 \mathrm{fs}$ can be achieved with a combination of Nd:glasses. The pulse shape can be optimized to a series of pulses in order to resonantly excite the plasma wave as proposed by $D$. Umstadter and E. Esarey. The laser system could be laser-diode-pumped Nd:glass and have an average power of $10 \mathrm{~kW}$. With an efficiency of about $10 \%$ the electrical power will be of the order of $100 \mathrm{~kW}$. With a few joules per pulse, gradients of $20 \mathrm{GeV} / \mathrm{m}$ could be produced. Such a system will be of the order of $100 \mathrm{~m}$ in length and could accelerate about $10^{10}$ electrons up to a fraction of a tera-electron-volt. 


\section{References}

1 D. Strickland and G. Mourou, Opt. Commun. 56, 219 (1985).

2 J. Zhou, G. Taft, C.-P. Huang, I. P. Christov, H. C. Kapteyn, and M. M. Murnane, Ultrafast Phenomena IX, P. F. Barbara, W. H. Knox, G. A. Mourou, and A. H. Zewail, eds. (Springer-Verlag, Berlin, 1994), p. 39; F. Krausz, Ch. Spielmann, P. F. Curley, T. Brabec, S. M. J. Kelly, A. Stingl, R. Szipöcs, E. Wintner, and A. J. Schmidt, ibid, p. 3.

3 T. Tajima and J. M. Dowson, Phys. Rev. Lett. 43, 267 (1979).

4 P. Sprangle, E. Esarey, A. Ting, and G. Joyce, Appl. Phys. Lett. 53, 2146 (1988).

5 D. Umstadter, E. Esarey, and J. Kim, Phys. Rev. Lett. 72, 1224 (1994).

6 C. Sauteret, D. Husson, G. Thiell, S. Seznec, S. Gary, A. Migus, and G. Mourou, Opt. Lett. 16, 238 (1991); C. Rouyer, E. Mazataud, I. Allais, A. Pierre, S. Seznec, C. Sauteret, G. Mourou, A. Migus, Opt. Lett. 18, 214 (1993).

7 M. Perry and G. Mourou, Science 264, 917 (1994).

8 D. Du, X. Liu, G. Korn, J. Squier, and G. Mourou, Appl. Phys. Lett. 64, 3071 (June 1994).

9 O. E. Martinez, IEEE J. Quantum Electron. 23, 1385 (1987).

10 M. Pessot, Opt. Commun. 62, 419 (1987).

11 J. Kmetec, J. J. Macklin, and J. F. Young, Opt. Lett. 16, 1001 (1991); A. Sullivan, H. Hamster, H. C. Kapteyn, S. Gordon, W. White, H. Nathel, R. J. Blair, R. W. Falcone, Opt. Lett. 16, 1406 (1991); T. Ditmire and M. D. Perry, Opt. Lett. 18, 426 (1993); P. Beaud, M. Richardson, E. Miesak, and B. T. Chai, Opt. Lett. 18, 1550 (1993); W. E. White, J. R. Hunter, L. Van Woerkom, T. Ditmire, and M. D. Perry, Opt. Lett. 17, 1067 (1992).

12 C. P. J. Barty, B. E. Lemoff, and C. L. Gordon III, Ultrafast Phenomena IX, P. F. Barbara, W. H. Knox, G. A. Mourou, and A. H. Zewail, eds. (Springer-Verlag, Berlin, 1994), p. 170.

13 J. Zhou, C. P. Huang, M. M. Murnane, and H. C. Kapteyn, to be published in Optics Letters (1994).

14 W.E. White,F.G. Patterson, R.ZL. Combs, D. F. Priceand R. L. Shepperd, Opt. Lett.. 18, 1343 (1994)

15 S. Kane, J. Squier, J. V. Rudd, and G. Mourou, Opt. Lett. 19, 1876 (1994).

16 T. Katsouleas, private communication. 
17 A. M. Weiner, D. E. Leaird, G. P. Weiderrecht, and K. A. Nelson, Science 247, 1317 (1990); D. Pinkos, J. Squier, B. Kohler, V. V. Yakovlev, K. R. Wilson, D. Schumacher, and P. H. Bucksbaum, Ultrafast Phenomena IX, P. F. Barbara, W. H. Knox, G. A. Mourou, and A. H. Zewail, eds. (SpringerVerlag, Berlin, 1994), p. 180.

18 Jeff Squier and Gerard Mourou, Laser Focus World 28, 51 (June 1992).

19 J. D. Kmetec, J. J. Macklin, and J. F. Young, Opt. Lett. 16, 1001 (1991).

20 K. Yamakawa, H. Sugio, H. Daido, M. Nakatsuka, Y. Kato, and S. Nakai, Opt. Commun. 112, 37 (1994).

21 B. J. Comaskey, R. Beach, G. Albrecht, W. J. Benett, B. L. Freitas, C. Petty, D. VanLue, D. Mundinger, and R. W. Solarz, IEEE J. Quantum Electron. 28, 992 (1992). 


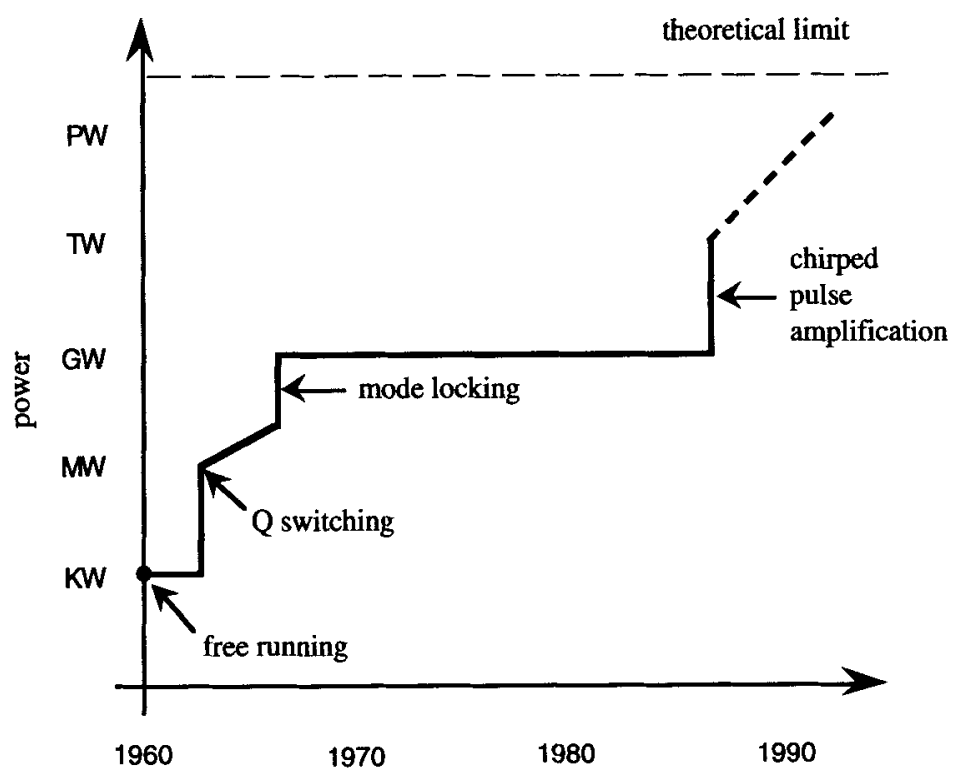

FIG. 1. Evolution of Laser Peak Power

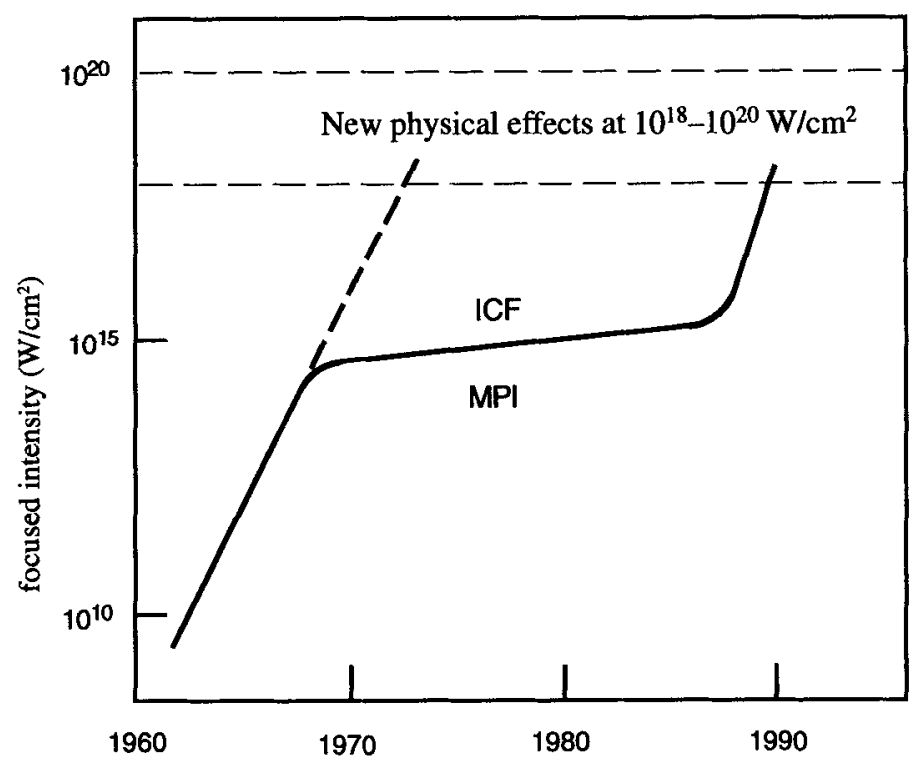

FIG. 2. History of Laser-Matter Interaction 


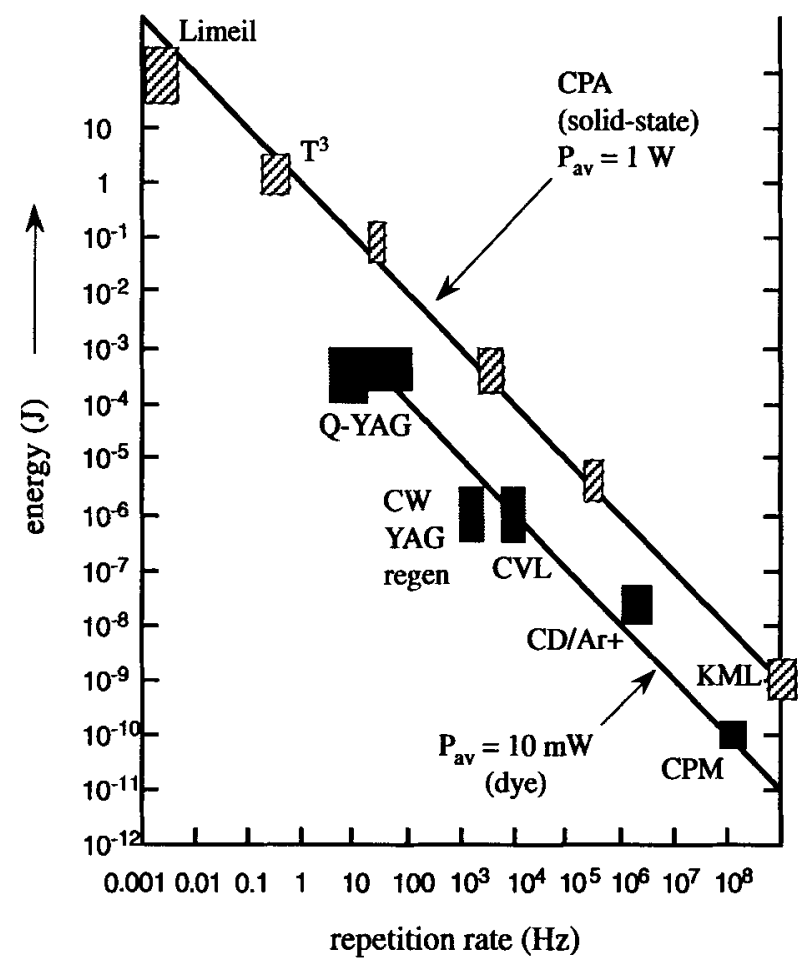

FIG. 3. Average Power of Femtosecond Lasers

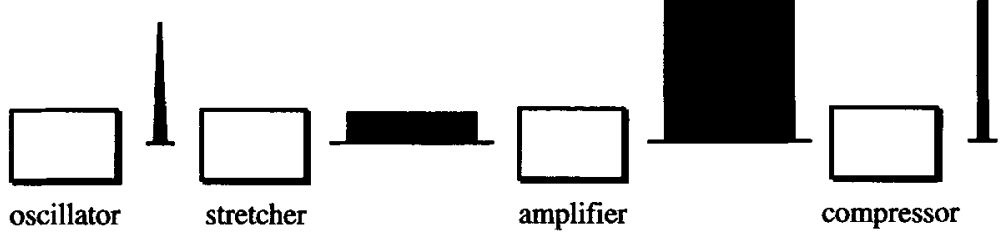

FIG. 4. Chirped Pulse Amplification 


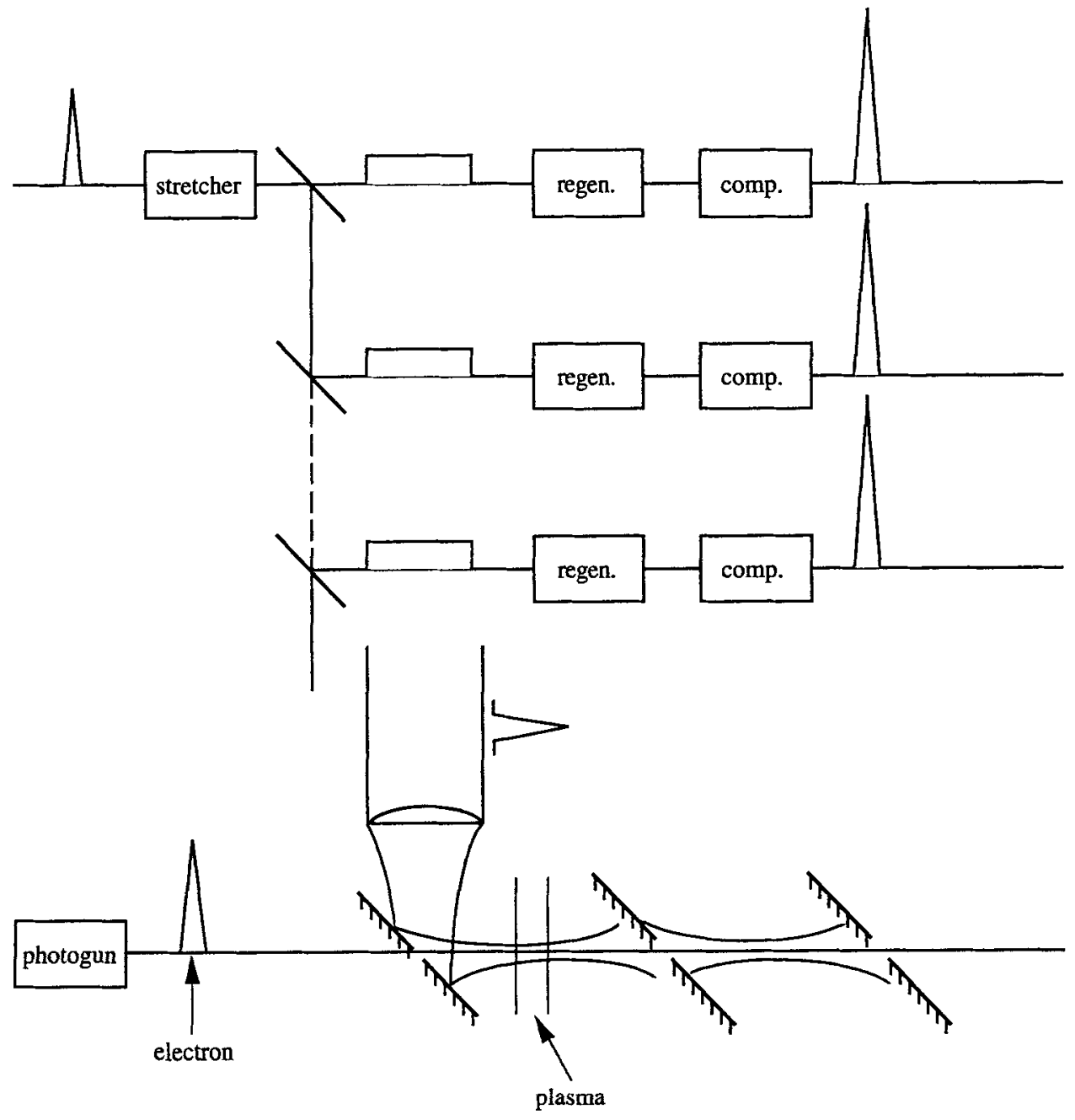

FIG. 5. High-Average-Power Wakefield Accelerator 